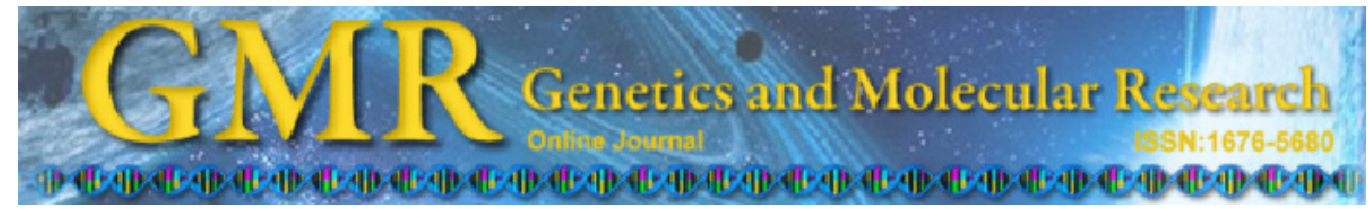

\title{
Position effect variegation and epigenetic modification of a transgene in a pig model
}

\author{
Z. Yin ${ }^{1 *}$, Q.R. Kong ${ }^{1 *}$, Z.P. Zhao ${ }^{2}$, M.L. Wu ${ }^{2}$, Y.S. Mu ${ }^{1}$ K. $\mathrm{Hu}^{1}$ and \\ Z.H. Liu ${ }^{1}$ \\ ${ }^{1}$ College of Life Science, Northeast Agricultural University of China, \\ Harbin, China \\ ${ }^{2}$ College of Animal Science and Technology, China Agricultural University, \\ Beijing, China \\ *These authors contributed equally to this study. \\ Corresponding author: Z.H. Liu \\ E-mail: liu086@yahoo.com
}

Genet. Mol. Res. 11 (1): 355-369 (2012)

Received July 7, 2011

Accepted November 11, 2011

Published February 16, 2012

DOI http://dx.doi.org/10.4238/2012.February.16.1

\begin{abstract}
Sequences proximal to transgene integration sites are able to regulate transgene expression, resulting in complex position effect variegation. Position effect variegation can cause differences in epigenetic modifications, such as DNA methylation and histone acetylation. However, it is not known which factor, position effect or epigenetic modification, plays a more important role in the regulation of transgene expression. We analyzed transgene expression patterns and epigenetic modifications of transgenic pigs expressing green fluorescent protein, driven by the cytomegalovirus (CMV) promoter. DNA hypermethylation and loss of acetylation of specific histone $\mathrm{H} 3$ and $\mathrm{H} 4$ lysines, except H4K16 acetylation in the CMV promoter, were consistent with a low level of transgene expression. Moreover, the degree of DNA methylation and histone $\mathrm{H} 3 / \mathrm{H} 4$ acetylation in the promoter region depended on the integration site; consequently, position effect variegation caused variations in epigenetic modifications. The transgenic pig fibroblast cell lines were treated with DNA methyltransferase inhibitor 5-Aza-2'-deoxycytidine and/or histone
\end{abstract}


deacetylase inhibitor trichostatin A. Transgene expression was promoted by reversing the DNA hypermethylation and histone hypoacetylation status. The differences in DNA methylation and histone acetylation in the CMV promoter region in these cell lines were not significant; however, significant differences in transgene expression were detected, demonstrating that variegation of transgene expression is affected by the integration site. We conclude that in this pig model, position effect variegation affects transgene expression.

Key words: PEV; DNA methylation; Histone acetylation; Transgene expression; Transgenic pig

\section{INTRODUCTION}

Transgenic technology has greatly facilitated our understanding of gene function, producing pharmaceutical proteins, and generating models for the study of human diseases. However, transgenes hardly express as people expected in transgenic animals and cell lines, and their expression level is predictable only to a limited extent (Houdebine, 2002). This could be mainly attributed to PEV (Gronbaek et al., 2007).

In PEV, host sequences proximal to the integration site can influence transgene expression in a positive or negative way. When a transgene is inserted into close proximity to heterochromatin such as telomere and centromere regions, its activity can become unstable, resulting in variations in expression level referred to as mosaicism or variegation (Eszterhas et al., 2002). Genomic DNA surrounding a well-expressed transgene favors the expression of another transgene when inserted into it, such as the ROSA26 locus in mouse (Strathdee et al., 2006; Irion et al., 2007). The phenomena which govern this effect seem rather complex. Subtle interactions between transgene and genomic DNA sequences seem to influence expression (Cranston et al., 2001). Thus, PEV is often an obstacle to obtaining transgene stable expression cell lines, and the same problem is common in the application of transgenic animals (Whitelaw et al., 2001). It has also been revealed that PEV appears to be the result of the spreading of a compacted chromatin state from heterochromatin to adjacent transgenes and the process is regulated by chromatin remodeling (Ebert et al., 2004, 2006).

Chromatin remodeling is thought to involve DNA methylation and, more importantly, histone acetylation. It is well established that DNA hypermethyaltion via forming compacted chromatin to prevent transcription factors binding can repress gene expression (Miranda and Jones, 2007). In DNA methylation, the repression is often brought about by methylation of $\mathrm{CpG}$ dinucleotides clustered to form $\mathrm{CpG}$ islands, which are usually located in the promoter (Esteller and Herman, 2002). Histone deacetylation at specific residues, mainly in histone H3 and $\mathrm{H} 4$, is also a suggested epigenetic modification, which suppresses gene expression by leading to chromatin condensation through interactions of the free lysine residues (Kouzarides, 2007). Recent studies reveal that regulation of histone acetylation can be functionally linked to reinforce the activation or repression of gene expression (Lee et al., 2007; Duncan et al., 2008; Li at al., 2008). Thus the level of the DNA methylation and histone acetylation in the promoter region can serve as an indicator of gene activity (Grassi et al., 2003).

An understanding of all the factors influencing transgene expression would be es- 
sential for effective production of transgenic animals and cell lines for various applications. In this study, we employed transgenic pigs produced by SCNT, expressing GFP driven by the CMV promoter, to check a position effect on transgene expression and analyze DNA methylation and histone acetylation marking on the CMV promoter. We observe that PEV may be mainly determined by an integration site involved with the variegation of DNA methylation and histone acetylation.

\section{MATERIAL AND METHODS}

\section{Establishment of transgenic pigs}

Fibroblast cells derived from E32 fetuses were transfected using the liposome-mediated plasmid pEGFP-C1 (Clontech) containing eGFP driven by the CMV promoter. Integration was based on a random insertion of the nonhomologous DNA vector into the host genome. After G418 selection, surviving cells were used as nuclear donors, and nuclear transfer was preformed as described (Liu et al., 2008). Positive transgenic pigs were identified by PCR using primers $\mathrm{P} 1$ and $\mathrm{P} 2$. The sequences of the primers were 5'-TGAACCGCATCGAGCT GAAGGG-3' (forward) and 5'-TCCAGCAGGACCATGTGATCGC-3' (reverse), and PCR generated a 308-bp product. All DNA samples were extracted using the Universal Genomic DNA Extraction Kit Ver. 3.0 (TaKaRa) according to manufacturer instructions.

\section{Transgene integration site analysis}

Thermal asymmetric interlaced (TAIL)-PCR, which relies on a series of PCR reactions with transgene specific and degenerate primers, was used for amplifying the site of integration of the transgene as described (Pillai et al., 2008). Junction PCR combined with transgene specific and integration site primers was performed to confirm the integration sites. The products of the third round of walking PCR were sequenced and analyzed by BLAST in the pig genome and EST database. The sequences of primers and detailed methods were described in the previous report (Kong et al., 2009a).

\section{Q-PCR analysis}

Total RNAs were extracted from each sample using the PureLink ${ }^{\mathrm{TM}}$ Micro-to-Midi system (Invitrogen) according to manufacturer instructions, and reverse transcription was used to generate cDNAs using PrimeScript ${ }^{\mathrm{TM}}$ RT Reagent Kit (TaKaRa). Real-time PCR was performed using SYBR Premix Ex Taq ${ }^{\mathrm{TM}}$ (TaKaRa) and the 7500 Real-Time PCR System (Applied Biosystems), with the following parameters: $95^{\circ} \mathrm{C}$ for $30 \mathrm{~s}$, followed by 40 two steps cycles at $95^{\circ} \mathrm{C}$ for $5 \mathrm{~s}$ and at $60^{\circ} \mathrm{C}$ for $34 \mathrm{~s}$. Primers for the GFP gene were $5^{\prime}$-TGAACCG CATCGAGCTGAAGGG-3' (forward) and 5'-ACCTTGATGCCGTTCTTCTGCTTG-3' (reverse). The $\beta$-actin gene was used as a reference gene and the primer sequences were $5^{\prime}$-AG ATCGTGCGGGACATCAAG-3' (forward) and 5'-GCGGCAGTGGCCATCTC-3' (reverse). The sizes of the amplification products were $110 \mathrm{bp}$ for the GFP gene, and $93 \mathrm{bp}$ for the $\beta$-actin gene. For each cDNA sample, both target and reference genes were always amplified independently in triplicate on the same plate and in the same experimental run. A melting curve 
analysis showed that all reactions were free of primer-dimers or other non-specific products (data not shown). $\mathrm{C}_{\mathrm{t}}$ values were calculated by the Sequence Detection System software (Applied Biosystems), and the amount of target sequence normalized to reference sequence was calculated as: $2^{-\Delta \Delta C t}$.

\section{Cell culture and treatments}

Fibroblast cells isolated from ear of the transgenic pigs were cultured and proliferated in DMEM $+20 \%$ FBS (Gibco). Cells were treated with media containing $0.25 \mu \mathrm{M}$ TSA (Sigma) for $24 \mathrm{~h}$ to inhibit histone deacetylation or $0.5 \mu \mathrm{M} 5$-aza-dC (Sigma) for $48 \mathrm{~h}$ to inhibit DNA methylation. After treatment, cells were harvested for the analysis of transgene expression, DNA methylation and histone acetylation.

\section{Flow cytometry}

The fluorescence intensities of the transgenic fibroblast cells were analyzed in an FACS Calibur (Becton-Dickinson). The argon laser was tuned to $488 \mathrm{~nm}$, and fluorescent cells were evaluated with a $525 \mathrm{~nm}$ band-pass filter. Dead cells and debris were excluded from the analysis by gating for intact cells using the forward and sideward scatter. Untransfected cells were always included as a control to detect autofluorescence.

\section{Bisulfite sequencing analysis of CMV promoter methylation}

Bisulfite modification was performed on $0.3 \mu \mathrm{g}$ of DNA from each sample using the EZ DNA Methylation-Gold ${ }^{\mathrm{TM}}$ Kit (Zymo research) according to the instruction manual. PCR primers were designed to amplify the CMV by MethPrimer software available on line (http:// www.urogene.org/methprimer/). The software was also used to predict $\mathrm{CpG}$ islands and $\mathrm{CpG}$ sites in the sequence. The following PCR primers could efficiently and specifically amplify a 278bp region containing one $\mathrm{CpG}$ island with $14 \mathrm{CpG}$ sites: gps: 5'-TGATTTTATGGGATTTTTT TATTTG-3' (forward) and gpa: 5'-ATTCACTAAACCAACTCTACTTATATAAAC-3' (reverse). None of these bisulfite dependent residues lie within the first six bp of the 3 '-end of the primers, so if some of these were not bisulfate-converted, this effect on the efficiency of the primer would be limited. The amplification of bisulfited-modified DNA was performed using Hot start Taq ${ }^{\mathrm{TM}}$ polymerase (TaKaRa) with the following conditions: $94^{\circ} \mathrm{C}$ for $5 \mathrm{~min}$, followed by 40 three step cycles at $94^{\circ} \mathrm{C}$ for $30 \mathrm{~s}, 52^{\circ} \mathrm{C}$ for $30 \mathrm{~s}$ and at $72^{\circ} \mathrm{C}$ for $1 \mathrm{~min}$. The PCR products were separated on $1 \%$ agarose gels and purified, followed by sequencing (Invitrogen). The presence of a cytosine residue after bisulfite treatment shows that the cytosine residue was protected from bisulfite modification by methylation. For each DNA sample, the number of protected cytosine residues was counted and converted to a percentage of the $14 \mathrm{CpG}$ cytosines present in the 278-bp region of the CMV that was analyzed. As a control, the pEGFP-C1 plasmid was treated and analyzed. At least ten clones were sequenced and analyzed for each sample.

\section{Chromatin immunoprecipitation analysis of histone acetylation on the CMV promoter}

Tissue and cell ChIP assays were carried out using the Acetyl-Histone Immunoprecip- 
itation (CHIP) Assay Kit (Millipore) with the following specific details or modifications. Cells were harvested and mixed with formaldehyde at a final concentration of $1.0 \%$ for $10 \mathrm{~min}$ at $37^{\circ} \mathrm{C}$ to cross-link protein to DNA. Cells were rinsed with cold PBS containing $1 \mathrm{mM}$ PMSF (Amresco) and resuspended in SDS lysis buffer. Lysates were sonicated using an Ultrasonic Homogenizer 4710 series to shear DNA into 200-1000 bp fragments. Protein-DNA complexes were immunoprecipitated using antibodies to acetyl-H3/H4 (Millipore), acetyl-K16 H4 (Millipore) and acetyl-K9 H3 (Millipore). Five $\mu \mathrm{L}$ of each specific antibody was added to lysates and incubated overnight at $4^{\circ} \mathrm{C}$. To reduce nonspecific background, immunocomplexes were blocked with salmon sperm DNA/Protein A Agarose Slurry by incubating for one hour at $4^{\circ} \mathrm{C}$ before chromatin immunoprecipitation. Beads were washed once with each of the following: low salt buffer, high salt buffer, $\mathrm{LiCl}$ buffer, and twice with TE buffer. Immunocomplexes were eluted by incubating beads at room temperature for $15 \mathrm{~min}$ in $250 \mu \mathrm{L}$ elution buffer $\left(0.1 \mathrm{M} \mathrm{NaHCO}_{3}, 1 \% \mathrm{SDS}\right)$, and the cross-links were reversed by incubating at $65^{\circ} \mathrm{C}$ for $4 \mathrm{~h}$. After incubation with $0.2 \mathrm{mg} / \mathrm{mL}$ RNase A at $37^{\circ} \mathrm{C}$ for $2 \mathrm{~h}$ and $0.2 \mathrm{mg} / \mathrm{mL}$ Proteinase $\mathrm{K}$ at $45^{\circ} \mathrm{C}$ for $1 \mathrm{~h}$, DNA was purified using the TOPure ${ }^{\mathrm{TM}} \mathrm{Gel} / \mathrm{PCR}$ Purification Kit (Gene Tech). Q-PCR was used to analyze the immunoprecipitated DNA as described above. Primers for the CMV promoter were 5'-CCTGGCATTATGCCCAGTACATGACC-3' (forward) and 5'-GGTG GAGACTTGGAAATCCCCGTG-3' (reverse), and the primers for the $\beta$-actin promoter, as a control, were 5'-TACCCACCCATTCGTCCTTTCATATTGC-3' (forward) and 5'-CCTCT TAGAGTCTGGTCCTGATTAGTTCGTG-3' (reverse). The sizes of the amplification products were $156 \mathrm{bp}$ for the CMV promoter and $150 \mathrm{bp}$ for the $\beta$-actin promoter. For each DNA sample, both CMV and control promoters were always amplified independently in triplicate on the same plate and in the same experimental run. The melting curve analysis showed that all reactions were free of primer-dimers or other non-specific products (data not shown). The $\mathrm{C}_{\mathrm{t}}$ value was calculated by the Sequence Detection System software (Applied Biosystems), and the amount of target normalized to reference was calculated by: $2^{-\Delta \Delta C t}$.

\section{Statistical analysis}

Statistical analysis was performed using SPSS 13.0 for MicroSoft ${ }^{\mathrm{TM}}$ Windows. Data are shown as mean $\pm \mathrm{SD}$. One-way ANOVA was used to assess differences between groups. Paired-Samples T Test was performed to assess the differences of GFP expression, DNA methylation and histone acetylation between two transgenic pigs in all tissues considered. The Duncan method was employed for pairwise comparison and followed by Bonferroni correction. $\mathrm{P}<0.05$ (two-tailed) was considered statistically significant.

\section{RESULTS}

\section{Generation of transgenic pigs}

A total of 1978 reconstructed embryos were transferred to ten recipients. Three recipients became pregnant and four founder GFP-positive transgenic pigs were born at full term. The overall efficiency of transgenic pig production was $0.69 \%$. Finally, two female pigs, named K25-2 and K25-3, survived to develop into adults (the others died at birth), and in the study, the two transgenic pigs were employed to analyze the expression and epigenetic modifications of the transgene. 


\section{Position effect on transgene expression}

The integration sites were cloned and determined by TAIL-PCR and junction PCR as described in a previous report (Kong et al., 2009a). The integration site in K25-2 is within an L1M LINE element, and it is known to be involved with retrotransferase expression. Due to the repetitive nature of these sequences, the L1M LINE element could not be deciphered, so it is not an active element (Shyman and Weaver, 1985; Loeb et al., 1986). That suggests a position effect on GFP expression and that the integration site may lead to transgene silencing. In K25-3, the integration site significantly matched the pig EST EV932945 (99\%) which is homologous with Aatf. This gene is expressed in oocytes, embryos, ear, brain, liver and so on (Gray et al., 2004; Cobellis et al., 2005; Bailey et al., 2006), so it appears that this region is transcriptionally active and could permit the transcription of transgenes.

By Q-PCR analysis, GFP mRNA expression was detected in all of the tissues studied for K25-2 and K25-3, namely intestine, ovary, lung, liver, tongue, kidney, heart, spleen, stomach, brain and ear (Figure 1). In K25-2, mRNA levels were significantly higher in liver, heart and stomach compared to ovary, tongue, spleen and brain $(\mathrm{P}<0.001)$. In K25-3, significantly higher levels of mRNA expression were detected in the liver, kidney, brain, spleen and ear compared to the intestine, ovary and lung $(\mathrm{P}<0.001)$. Considering all tissues, the difference of GFP mRNA expression between the two transgenic pigs was not significant $(\mathrm{P}=0.414)$; however, we found that in most tissues, except in ovary $(\mathrm{P}=0.063)$, the GFP mRNA levels between K25-2 and K25-3 were significantly different $(\mathrm{P}<0.001)$, indicating a position effect on transgene expression. In general, expression and epigenetic modifications are functionally related, so expression variegation between the two transgenic pigs could be also due to the spread of the epigenetic modifications marking on the promoter of the transgene (Grassi, 2003). Therefore, we analyzed the DNA methylation and histone acetylation of the CMV promoter in the two transgenic pigs.

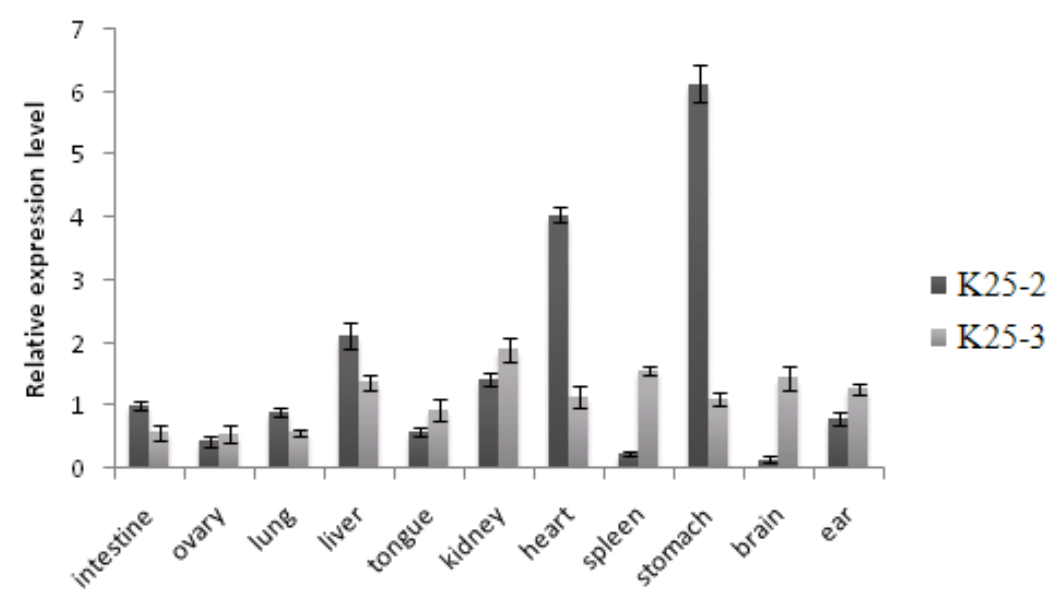

Figure 1. Variegation of GFP expression in various tissues of transgenic pigs. GFP mRNA expression levels were detected by Q-PCR analysis in various tissues of K25-2 and K25-3. Significant differences of GFP mRNA expression were shown in the tissues of K25-2 $(\mathrm{P}<0.001)$ and $\mathrm{K} 25-3(\mathrm{P}=0.016)$. In most tissues, except in ovary $(\mathrm{P}=0.063)$, the differences between K25-2 and K25-3 were significant $(\mathrm{P}<0.001)$. Error bars denote standard deviations. 


\section{Differential methylation of CMV promoter}

Transgene expression may be repressed by DNA methylation (Grassi, 2003). Therefore, the methylation status of a 278-bp region in the CMV promoter containing one $\mathrm{CpG}$ island with $14 \mathrm{CpG}$ sites in various tissues of K25-2 and K25-3 was examined (Figure 2). The bisulfite sequencing method is able to reveal the methylation status of all the cytosine residues in a DNA region of interest (Grunau et al., 2001). In the study, the pEGFP-C1 plasmid was treated by bisulfite modification and analyzed as a control. On average, $99 \%$ of the cytosine residues in the plasmid were converted to thymidine, indicating that the bisulfite conversion reaction on other samples was at least $99 \%$ efficient.

$\mathrm{K} 25-2$

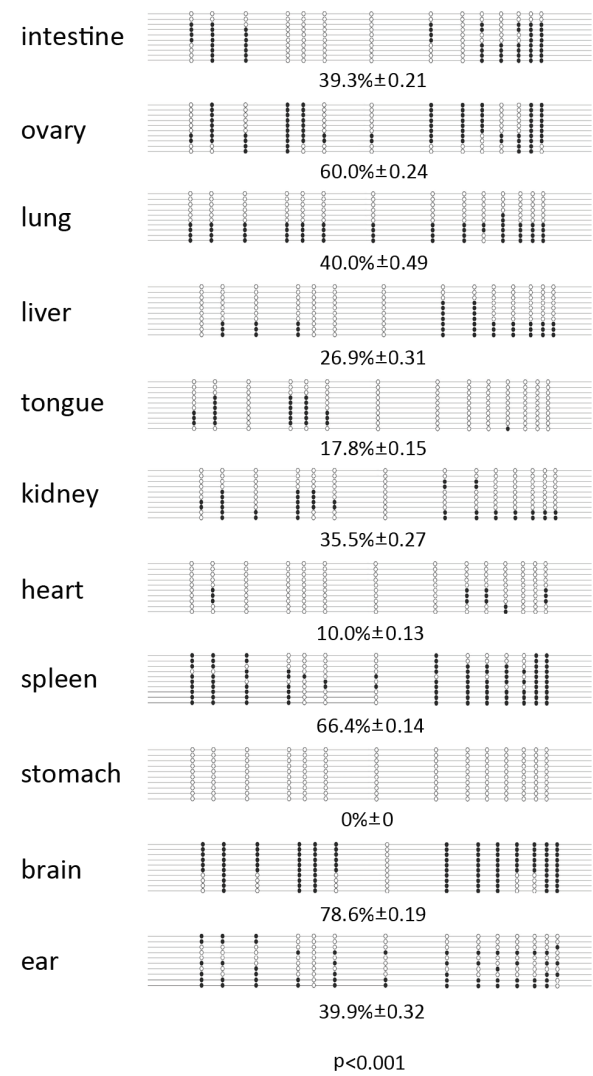

K25-3

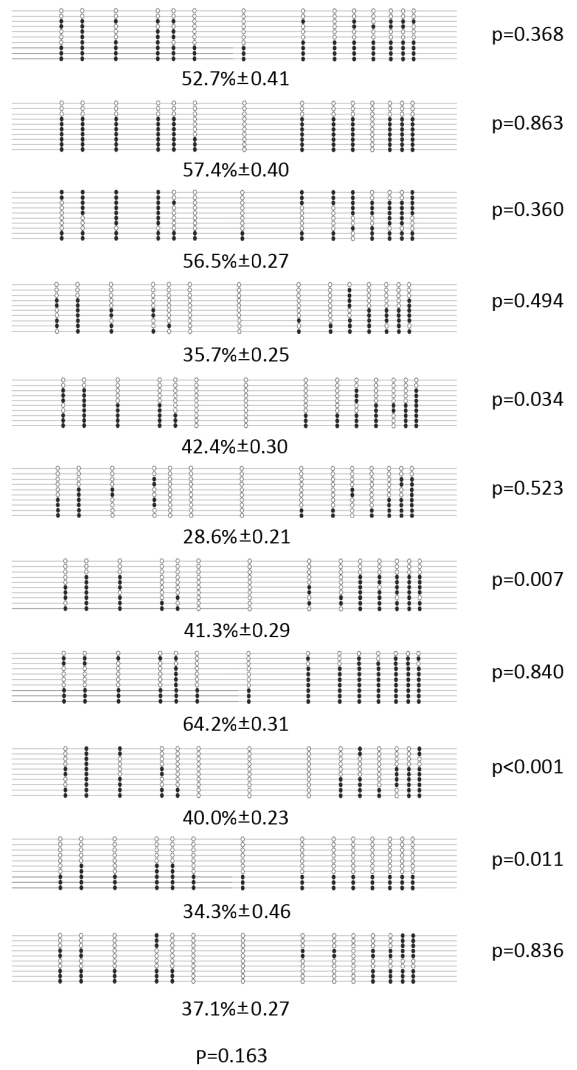

Figure 2. Variegation of CMV promoter methylation in various tissues of transgenic pigs. CMV promoter methylation status in various tissues of K25-2 and K25-3 was shown by the bisulfite sequencing method. The methylation status of a 278-bp region of the CMV promoter containing one $\mathrm{CpG}$ island with $14 \mathrm{CpG}$ sites was examined. Methylated and non-methylated $\mathrm{CpGs}$ dinucleotides of each clone are illustrated with closed and open circles, respectively. The variegations of CMV methylation status were observed. The difference in K25-2 was significant $(\mathrm{P}<0.001)$, but in $\mathrm{K} 25-3(\mathrm{P}=0.163)$ was not. The differences of $\mathrm{CMV}$ methylation in these tissues between K25-2 and K25-3 were observed and the differences in tongue $(\mathrm{P}=0.034)$, heart $(\mathrm{P}=0.007)$, stomach ( $\mathrm{P}$ $<0.001)$ and brain $(\mathrm{P}=0.011)$ were significant. 
Variegations of CMV methylation were observed in various tissues of K25-2 and K25-3. The difference in K25-2 was significant $(\mathrm{P}<0.001)$, but in $\mathrm{K} 25-3(\mathrm{P}=0.163)$ it was not. In K25-2, there was a hypermethylation status in ovary $(60.0 \%)$, spleen (66.4\%) and brain (78.6\%), and a hypomethylation status in liver (26.9\%), tongue (17.8\%), kidney $(35.5 \%)$ and heart $(10.0 \%)$. Moreover, the CMV promoter was in an unmethylated status in stomach of K25-2 (0\%). While, in K25-3, the intestine (52.7\%), ovary (57.4\%), lung $(56.5 \%)$ and spleen $(64.2 \%)$ were in a hypermethylation status, and the liver $(35.7 \%)$, kidney $(28.6 \%)$, brain $(34.3 \%)$ and ear $(37.1 \%)$ were in a hypomethylation status. Though, on the whole, the CMV methylation levels between K25-2 and K25-3 were not significantly different $(\mathrm{P}=0.325)$, the differences of $\mathrm{CMV}$ methylation in tongue $(\mathrm{P}=0.034)$, heart $(\mathrm{P}=0.007)$, stomach $(\mathrm{P}<0.001)$ and brain $(\mathrm{P}=0.011)$ between $\mathrm{K} 25-2$ and $\mathrm{K} 25-3$ were significant and could have resulted from a position effect on transgene methylation. In the study, except in tongue of K25-2 and in spleen of K25-3, a hypermethylation status of CMV promoter corresponded to a low level of transgene expression, similarly, a hypomethylation status corresponding to a high level of expression, indicating that transgene expression is associated with promoter methylation status.

\section{Histone acetylation on CMV promoter}

In general, increased histone acetylation is consistent with increased gene activation and vice versa (Jenuwein and Allis, 2001). Therefore, the enrichments of acetyl-H3/H4, acetyl-K9 H3 (referred to as an activating mark) and acetyl-K16 H4, in the CMV promoter region in various tissues of K25-2 and K25-3 were quantified by chromatin immunoprecipitation (ChIP) analysis (Figure 3).

Significant differences of histone acetylation at the CMV promoter were observed in various tissues of K25-2 and K25-3. As expected, tissues expressing high levels of GFP have a histone acetylation pattern at the CMV promoter consistent with active expression. Specifically, transgene expression was associated with the levels of H3 (Figure 3A), H3K9 (Figure 3B) and H4 (Figure 3C) acetylation relative to the modification levels measured at the active $\beta$-actin promoter, but no close correlation of transgene expression with H4K16 acetylation was observed (Figure 3D). Furthermore, on all tissues considered, the differences of histone acetylation in H3, H3K9, H4 and H4K16 were not significant between K25-2 and K25-3 ( P = 0.680, 0.516, 0.944 and 0.339 , respectively); however, in most cases, the differences of histone acetylation between the same tissue of the two transgenic pigs were significant $(\mathrm{P}<0.05)$, except acetyl-H3 in ovary $(\mathrm{P}=0.874)$, acetyl-H4 in heart $(\mathrm{P}=0.612)$, acetyl-K16 H4 in ovary $(\mathrm{P}=0.901)$ and spleen $(\mathrm{P}=0.792)$. Differences of H3K9 acetylation were significant in all the tissues between $\mathrm{K} 25-2$ and $\mathrm{K} 25-3(\mathrm{P}<0.001)$, suggesting that H3K9 acetylation could play a decisive role in the regulation of transgene expression. These results reveal that DNA hypermethylation and loss of acetylation of specific histone $\mathrm{H} 3$ and $\mathrm{H} 4$ lysines, except H4K16 acetylation, in the CMV promoter are consistent with the low level of transgene expression. Moreover, transgene expression and promoter epigenetic modifications are associated with the integration site, and a position effect is involved with variegation of epigenetic modifications. 

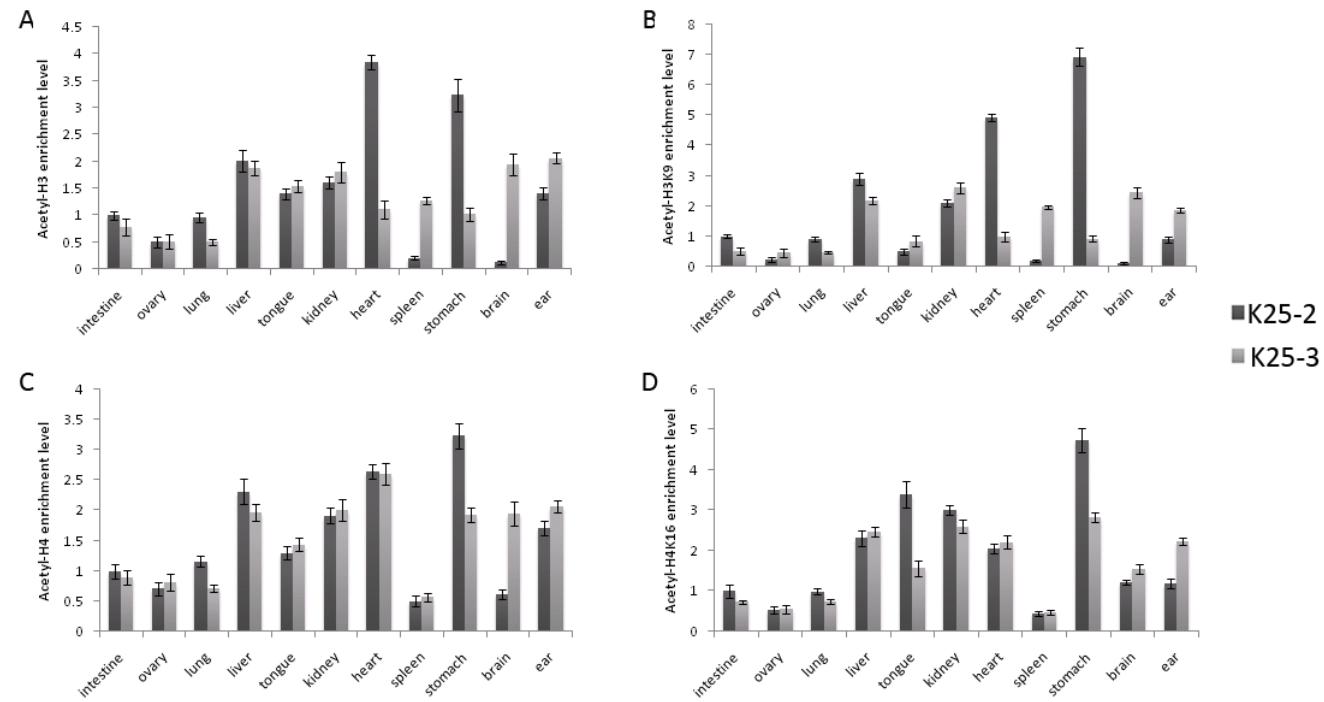

Figure 3. Variegation of histone acetylation at the CMV promoter in various tissues of transgenic pigs. A. ChIP analysis measuring acetylated histone $\mathrm{H} 3$ marking at the CMV promoter in various tissues of K25-2 and $\mathrm{K} 25-$ 3. On all tissues considered, the difference between $\mathrm{K} 25-2$ and $\mathrm{K} 25-3$ was not significant $(\mathrm{P}=0.680)$; however, except acetyl-H3 in ovary $(\mathrm{P}=0.874)$, the differences in other tissues were significant $(\mathrm{P}<0.05)$. B. ChIP analysis measuring acetylated histone H3K9 marking at the CMV promoter in various tissues of K25-2 and K25-3. Though, on all tissues considered, the difference between K25-2 and K25-3 was not significant $(\mathrm{P}=0.516)$, the differences in these tissues between K25-2 and K25-3 were significant $(\mathrm{P}<0.001)$. C. ChIP analysis measuring acetylated histone $\mathrm{H} 4$ at the CMV promoter in various tissues of K25-2 and K25-3. On all tissues considered, the difference between K25-2 and K25-3 was not significant $(\mathrm{P}=0.944)$; however, except acetyl-H4 in heart $(\mathrm{P}=0.612)$, the differences in other tissues were significant $(\mathrm{P}<0.05)$. D. ChIP analysis measuring acetylated histone H4K16 at the CMV promoter in various tissues of K25-2 and K25-3. On all tissues considered, the difference between K25-2 and K25-3 was not significant $(\mathrm{P}=0.339)$; however, except acetyl-K16 H4 in ovary $(\mathrm{P}=0.901)$ and spleen $(\mathrm{P}=0.792)$, the differences in other tissues were significant $(\mathrm{P}<0.05)$; Immunoprecipitated DNA levels were quantified by Q-PCR relative to the modification levels measured at the active $\beta$-actin promoter. Error bars denote standard deviations.

\section{5-Aza-dC and/or TSA treatment regulate transgene expression and epigenetic modification}

One of the hallmarks of epigenetic modification is reversible (Reik, 2007). In order to check which factor, position effect or epigenetic modification is more significant in the determination of transgene expression, we employed 5-Aza-dC and TSA to treat fibroblast cell lines of K25-2 and K25-3, expecting the treatment would be able to reverse the epigenetic modifications of the CMV promoter, and then test the position effect on transgene expression.

Changes in GFP mRNA level and rate of GFP expressing cells were measured in fibroblast cell lines of K25-2 and K25-3 after 5-Aza-dC and/or TSA treatment by Q-PCR (Figure 4A) and flow cytometry analysis (Figure 4B). Significant increases of transgene expression were observed in both cell lines after 5-Aza-dC and/or TSA treatment $(\mathrm{P}<0.001)$. Specifically, combining treatment with 5-Aza-dC and TSA induced the most apparent increase. DNA methylation levels at the CMV promoter in two cell lines after 5-Aza-dC and/or TSA treatment were measured by bisulfate sequencing (Figure 5). As expected, the CMV methyla- 
A

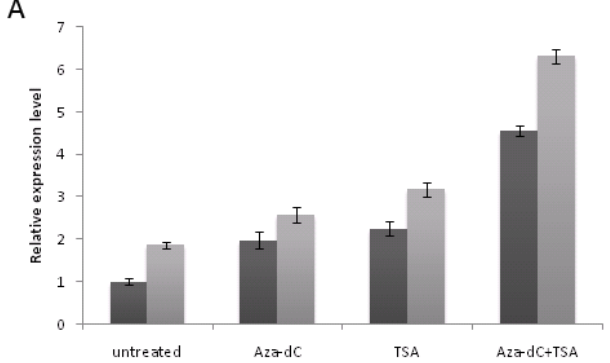

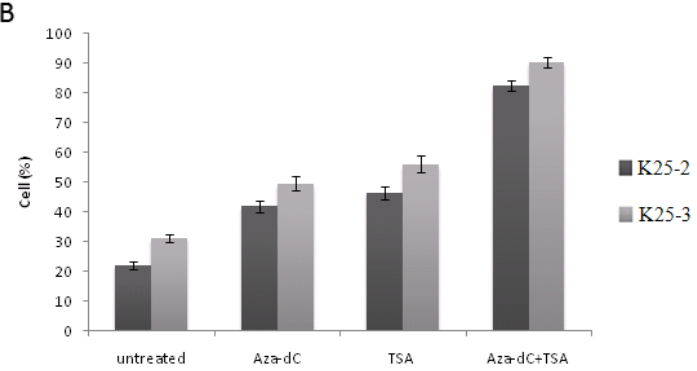

Figure 4. GFP expression in fibroblast cell lines of transgenic pigs after 5-Aza-dC and/or TSA treatment. GFP expression was detected by Q-PCR and flow cytometry analysis in fibroblast cell lines of K25-2 and K25-3 after 5-Aza-dC and/or TSA treatment. (A) Inhibition of DNA methylation and histone deacetylation significantly increased GFP mRNA levels in transgenic cell lines of K25-2 and K25-3 ( $<<0.001)$; (B) The rate of GFP positive cells in transgenic cell lines increased with significant differences by inhibition of DNA methylation and histone deacetylation $(\mathrm{P}<0.001)$. Combination treatment with 5-Aza-dC and TSA simultaneously resulted in a synergistic induction of GFP expression with a more significant increase compared to either treatment alone in both cell lines. Error bars denote standard deviations.

tion levels were decreased markedly, and the treatment by 5-Aza-dC, DNA methyltransferase inhibitor, revealed significantly reduced levels in both cell lines $(\mathrm{P}<0.001)$. Corresponding to the increased expression, the levels of histone acetylations enrichment at the CMV promoter increased significantly after 5-Aza-dC and/or TSA treatment $(\mathrm{P}<0.001)$, except for $\mathrm{H} 4 \mathrm{~K} 16$ acetylation that showed little response to 5-Aza-dC treatment $(\mathrm{P}=0.136$ in K25-2; $\mathrm{P}$ $=0.819$ in K25-3). Specifically, the treatment by TSA, histone deacetylase inhibitor, induced a greater increase in acetylation level. From the results, we also found DNA methylation was sensitive to both 5-Aza-dC and TSA treatment, though more sensitive to 5-Aza-dC treatment; however, in contrast, histone acetylation only showed a sensitive response to TSA treatment but little response to 5-Aza-dC treatment, suggesting TSA had a dual function in epigenetic regulation. Moreover, in the study, there was no significantly higher GFP mRNA level detected after TSA treatment compared to 5-Aza-dC treatment in the K25-2 cell line (P $=0.074)$; however, a significantly higher expression level was found in the K25-3 cell line (P $<0.001)$. That may be due to the higher methylation level at the CMV promoter in the K25-2 cell line and stronger response to histone deacetylase inhibitor treatment in the K25-3 cell line (Figure 6).

Combining treatment with 5-Aza-dC and TSA simultaneously resulted in a synergistic induction of GFP expression with a more significant increase compared to either treatment on its own in both cell lines and the CMV promoter status of DNA hypermethylation and histone hypoacetylation was significantly reversed $(\mathrm{P}<0.001)$, suggesting at best an additive effect on transgene expression and epigenetic regulation. Furthermore, after the combination treatment, DNA methylation and enrichment of histone acetylations at the CMV promoter, except H4K16 acetylation, reached a similar level without a significant difference $(\mathrm{P}>0.05)$, and the difference of transgene expression level between K25-2 and K25-3 cell lines declined from approximately 1 -fold to 0.3 -fold in mRNA level and 0.5 -fold to 0.1 -fold in rate of GFP expressing cells; however, the expression difference was still significant $(\mathrm{P}<0.001)$, suggesting transgene expression level could be mainly regulated by a position effect. 


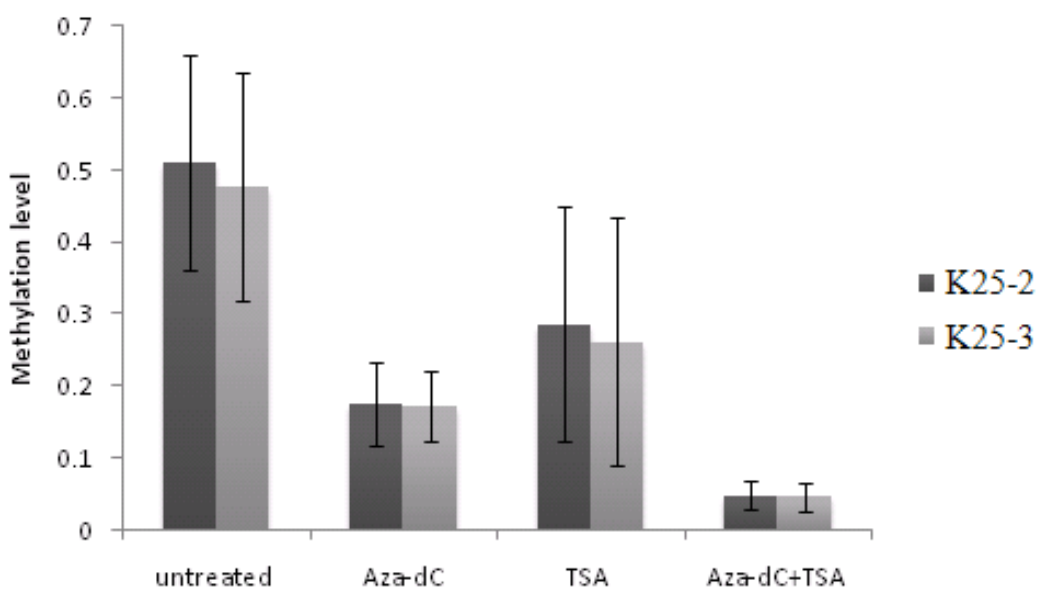

Figure 5. Methylation status of the CMV promoter in fibroblast cell lines of transgenic pigs after 5-Aza-dC and/ or TSA treatment. CMV promoter methylation status in fibroblast cell lines of K25-2 and K25-3 was shown by the bisulfite sequencing method after 5-Aza-dC and/or TSA treatment and the CMV methylation levels were decreased markedly. The treatment by 5-Aza-dC alone or the combination of two agents revealed significantly reduced levels in these cell lines $(\mathrm{P}<0.001)$. Furthermore, the CMV promoter methylation status showed no significant difference between the two cell lines after the combination treatment $(\mathrm{P}=0.911)$. Error bars denote standard deviations.

A

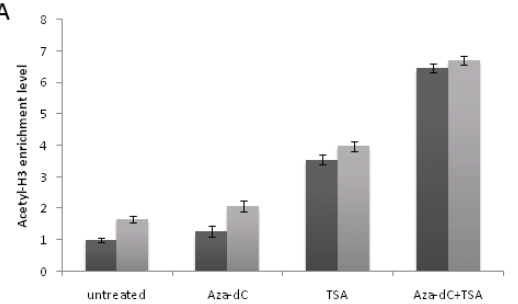

C

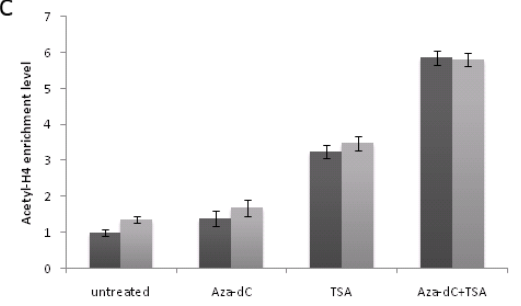

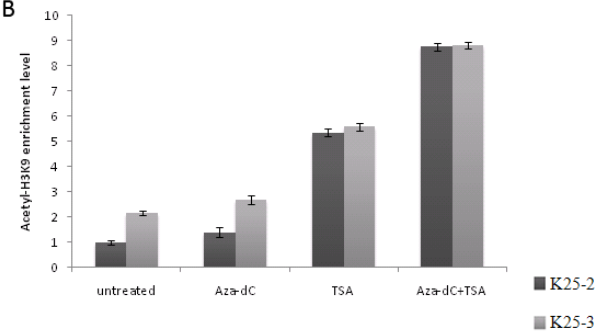

D

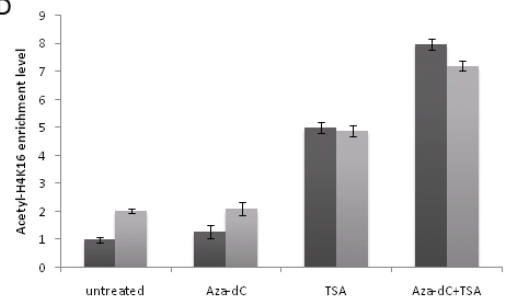

Figure 6. Histone modifications at the CMV promoter in fibroblast cell lines of transgenic pigs after 5-Aza-dC and/or TSA treatment. A to D: ChIP analysis measuring acetylated histone H3, H3K9, H4 and H4K16 marking at the CMV promoter in fibroblast cell lines of K25-2 and K25-3 after 5-Aza-dC and/or TSA treatment. The levels of histone acetylations enrichment at the CMV promoter were significant increased after 5-Aza-dC treatment $(\mathrm{P}<$ 0.001 ), except H4K16 acetylation ( $\mathrm{P}=0.136$ in $\mathrm{K} 25-2$ and $\mathrm{P}=0.819$ in $\mathrm{K} 25-3$ ). By TSA alone or the combination of two agents significant increases were observed in all these histone modifications $(\mathrm{P}<0.001)$ and the combination treatment induced more markedly. Except for acetyl-H4K16 $(\mathrm{P}<0.001)$, the acetylation enrichment showed no significant difference between the two cell lines after the combination treatment $(\mathrm{P}=0.137$ in acetyl-H3, $\mathrm{P}=0.906$ in acetyl-H3K9 and $\mathrm{P}=0.712$ in acetyl-H4). Error bars denote standard deviations. 


\section{DISCUSSION}

To our knowledge, this is the first study to systematically analyze the effect of a position effect and promoter epigenetic modifications on transgene expression in large domestic animal models. We presented transgene expression pattern and epigenetic analysis of two transgenic pigs with GFP gene integrated into different integration sites under the control of the CMV promoter.

Previous reports have demonstrated the variegation of transgene expression in different tissues of transgenic animals (Villuendas et al., 2001; Yang et al., 2008), and the phenomenon was further evident in our study. The variegation in transgene expression is attributed to either copy number effect or, more importantly, a position effect (Kaufman et al., 2008). In our previous report, 18.87, 30.58 copies respectively in K25-2 and K25-3 have been detected by absolute quantitative real-time PCR and the copy number effect has been demonstrated (Kong et al., 2009b). In this study, the position effect was mainly checked.

Sequences proximal to transgene integration sites are able to regulate transgene expression resulting in complex PEV (Clark et al., 1994). Previous experimental data have revealed the view that enhancers and silencers from neighbor genes are supposed to activate or inactivate transgenes, and some insulators have been shown to stimulate transgene expression by preventing the local formation of inactive heterochromatin (Houdebine, 2002). In this study, the transgene integration sites in K25-2 and K25-3 were checked and single different integration sites were detected in the two transgenic pigs. It is consistent with previous reports that when the integration is within a permissive region as in K25-3, the transgene can be actively transcribed (Cranston et al., 2001). While, interestingly, in K25-2, the integration is in an L1M LINE element where the transgene should be repressed (Mehta et al., 2009); however, we observed transgene expression in all tissues detected. Furthermore, we found the GFP expression levels between K25-2 and K25-3 were varied in most tissues. We suppose PEV may be related to the different integration sites in the two transgenic pigs.

In addition, PEV is often associated with variegation of epigenetic modifications. In the study, we observed that position effect is involved with epigenetic markings at the CMV promoter including DNA methylation and histone acetylation. Consistent with previous reports, hypomethylation corresponded to a high level of transgene expression in all the tissues detected of K25-2 and K25-3 and vice versa (Tshuikina et al., 2008). Moreover, variegations of CMV methylation in most tissues between K25-2 and K25-3 were observed and could be due to a position effect. It is proposed that DNA methylation is not always sufficient to regulate expression, but interaction with transcription repressors and/or repressive chromatin organization is also required. In general, histone acetylation is mainly associated with the formation of active chromatin structure and promoting the binding of chromatin-associated proteins. Deacetylation of histone $\mathrm{H} 3$ and $\mathrm{H} 4$ lysines are critically involved in the silencing of transgenes (Chen and Townes, 2000). In the study, the enrichments of acetyl-H3/H4, acetyl$\mathrm{K} 9 \mathrm{H} 3$ and acetyl-K16 H4, in the CMV promoter region, in various tissues of $\mathrm{K} 25-2$ and $\mathrm{K} 25-$ 3 were checked. Consistent with previous reports, transgene expression tightly correlated with $\mathrm{H} 3, \mathrm{H} 3 \mathrm{~K} 9$ and $\mathrm{H} 4$ acetylation, in contrast, H4K16 acetylation did not show the point, which is consistent with the previous points that acetyl-K16 H4 could regulate gene expression by a dual mechanism, disrupting formation of higher-order chromatin structure and changing the binding of chromatin-associated proteins (Shogren-Knaak et al., 2006). Variegations of 
histone acetylation at the CMV promoter in most tissues between K25-2 and K25-3 were observed, indicating a position effect on histone acetylation.

The above results reveal that transgene expression is regulated by both position effect and epigenetic modification, while epigenetic modifications are involved with position effect. However, which factor, position effect or epigenetic modification is more significant in the determination of transgene expression is unclear. In order to check the point, transgene expression and promoter epigenetic modification were checked in fibroblast cell lines of K25-2 and K25-3 after 5-Aza-dC and/or TSA treatment. A significant increase of transgene expression in both cell lines was induced after treatment. Furthermore, we also found DNA methylation was sensitive to both 5-Aza-dC and TSA treatment, though more sensitive to 5-Aza-dC treatment. In contrast, histone acetylation only showed a sensitive response to TSA treatment but little response to 5-Aza-dC treatment, revealing the dual function of TSA to epigenetic regulation. These results demonstrate that DNA methyltransferase inhibitor 5-Aza-dC and histone deacetylase inhibitor TSA can promote transgene expression by reversing the DNA hypermethylation and histone hypoacetylation status. The combination treatment with both agents resulted in a synergistic activation of the transgene and reversion of DNA hypermethylation and histone hypoacetylation at the CMV promoter, suggesting a cross talk between histone acetylation and DNA demethylation. However, the difference in transgene expression level between K25-2 and K25-3 cell lines was still significant, while DNA methylation and enrichment of histone acetylations at the CMV promoter, except H4K16 acetylation, were reversed to a similar level without significant difference, so we propose a position effect may be mainly responsible for the difference of transgene expression between K25-2 and K25-3 cell lines and that could be contributed to by enhancers, silencers and insulators proximal to the integration sites (Eszterhas et al., 2002).

\section{CONCLUSIONS}

In this study, we employed two transgenic pigs expressing GFP under the control of the CMV promoter to analyze a position effect and epigenetic modifications on the regulation of transgene expression. We found that the position effect is associated with integration sites, and can control transgene expression directly by regulatory elements, such as enhancers and silencers, and indirectly by epigenetic modifications. In summary, our results provide a clear demonstration that PEV may be mainly determined by integration sites involved with the variegation of epigenetic modifications.

\section{ACKNOWLEDGMENTS}

The authors gratefully acknowledge our colleagues in the "Lab of Embryo Biotechnology" for helpful discussion. Research supported by a grant from the State Transgenic Research Program of China (\#2008ZX08006-002) (\#2009ZX08006-001B) and Innovative Research Team of NEAU.

\section{REFERENCES}

Bailey PJ, Klos JM, Andersson E, Karlen M, et al. (2006). A global genomic transcriptional code associated with CNSexpressed genes. Exp. Cell Res. 312: 3108-3119. 
Chen WY and Townes TM (2000). Molecular mechanism for silencing virally transduced genes involves histone deacetylation and chromatin condensation. Proc. Natl. Acad. Sci. U. S. A. 97: 377-382.

Clark AJ, Bissinger P, Bullock DW, Damak S, et al. (1994). Chromosomal position effects and the modulation of transgene expression. Reprod. Fertil. Dev. 6: 589-598.

Cobellis G, Nicolaus G, Iovino M, Romito A, et al. (2005). Tagging genes with cassette-exchange sites. Nucleic Acids Res. 33: e44.

Cranston A, Dong C, Howcroft J and Clark AJ (2001). Chromosomal sequences flanking an efficiently expressed transgene dramatically enhance its expression. Gene 269: 217-225.

Duncan EM, Muratore-Schroeder TL, Cook RG, Garcia BA, et al. (2008). Cathepsin L proteolytically processes histone H3 during mouse embryonic stem cell differentiation. Cell 135: 284-294.

Ebert A, Schotta G, Lein S, Kubicek S, et al. (2004). Su(var) genes regulate the balance between euchromatin and heterochromatin in Drosophila. Genes Dev. 18: 2973-2983.

Ebert A, Lein S, Schotta G and Reuter G (2006). Histone modification and the control of heterochromatic gene silencing in Drosophila. Chromosome Res. 14: 377-392.

Esteller M and Herman JG (2002). Cancer as an epigenetic disease: DNA methylation and chromatin alterations in human tumours. J. Pathol. 196: 1-7.

Eszterhas SK, Bouhassira EE, Martin DI and Fiering S (2002). Transcriptional interference by independently regulated genes occurs in any relative arrangement of the genes and is influenced by chromosomal integration position. Mol. Cell Biol. 22: 469-479.

Grassi G, Maccaroni P, Meyer R, Kaiser H, et al. (2003). Inhibitors of DNA methylation and histone deacetylation activate cytomegalovirus promoter-controlled reporter gene expression in human glioblastoma cell line U87. Carcinogenesis 24: 1625-1635.

Gray PA, Fu H, Luo P, Zhao Q, et al. (2004). Mouse brain organization revealed through direct genome-scale TF expression analysis. Science 306: 2255-2257.

Gronbaek K, Hother C and Jones PA (2007). Epigenetic changes in cancer. APMIS 115: 1039-1059.

Grunau C, Clark SJ and Rosenthal A (2001). Bisulfite genomic sequencing: systematic investigation of critical experimental parameters. Nucleic Acids Res. 29: E65.

Houdebine LM (2002). The methods to generate transgenic animals and to control transgene expression. J. Biotechnol. 98: $145-160$.

Irion S, Luche H, Gadue P, Fehling HJ, et al. (2007). Identification and targeting of the ROSA26 locus in human embryonic stem cells. Nat. Biotechnol. 25: 1477-1482.

Jenuwein T and Allis CD (2001). Translating the histone code. Science 293: 1074-1080.

Kaufman WL, Kocman I, Agrawal V and Rahn HP (2008). Homogeneity and persistence of transgene expression by omitting antibiotic selection in cell line isolation. Nucleic Acids Res. 36: e111.

Kong Q, Wu M, Zhu J and Bou G (2009a). Transgene copy number and integraton site analysis in transgenic pig. Prog. Biochem. Biophys. 36: 1617-1625.

Kong Q, Wu M, Huan Y, Zhang L, et al. (2009b). Transgene expression is associated with copy number and cytomegalovirus promoter methylation in transgenic pigs. PLoS One 4: e6679.

Kouzarides T (2007). Chromatin modifications and their function. Cell 128: 693-705.

Lee JS, Shukla A, Schneider J, Swanson SK, et al. (2007). Histone crosstalk between H2B monoubiquitination and H3 methylation mediated by COMPASS. Cell 131: 1084-1096.

Li F, Huarte M, Zaratiegui M, Vaughn MW, et al. (2008). Lid2 is required for coordinating H3K4 and H3K9 methylation of heterochromatin and euchromatin. Cell 135: 272-283.

Liu ZH, Song J, Wang ZK, Tian JT, et al. (2008). Green fluorescent protein (GFP) transgenic pig produced by somatic cell nuclear transfer. Chin. Sci. Bull. 53: 1035-1039.

Loeb DD, Padgett RW, Hardies SC, Shehee WR, et al. (1986). The sequence of a large L1Md element reveals a tandemly repeated 5' end and several features found in retrotransposons. Mol. Cell Biol. 6: 168-182.

Mehta AK, Majumdar SS, Alam P, Gulati N, et al. (2009). Epigenetic regulation of cytomegalovirus major immediateearly promoter activity in transgenic mice. Gene 428: 20-24.

Miranda TB and Jones PA (2007). DNA methylation: the nuts and bolts of repression. J. Cell Physiol. 213: 384-390.

Pillai MM, Venkataraman GM, Kosak S and Torok-Storb B (2008). Integration site analysis in transgenic mice by thermal asymmetric interlaced (TAIL)-PCR: segregating multiple-integrant founder lines and determining zygosity. Transgenic Res. 17: 749-754.

Reik W (2007). Stability and flexibility of epigenetic gene regulation in mammalian development. Nature 447: 425-432.

Shogren-Knaak M, Ishii H, Sun JM, Pazin MJ, et al. (2006). Histone H4-K16 acetylation controls chromatin structure and protein interactions. Science 311: 844-847. 
Shyman S and Weaver S (1985). Chromosomal rearrangements associated with LINE elements in the mouse genome. Nucleic Acids Res. 13: 5085-5093.

Strathdee D, Ibbotson H and Grant SG (2006). Expression of transgenes targeted to the Gt(ROSA)26Sor locus is orientation dependent. PLoS One 1: e4.

Tshuikina M, Nilsson K and Oberg F (2008). Positive histone marks are associated with active transcription from a methylated ICSBP/IRF8 gene. Gene 410: 259-267.

Villuendas G, Gutierrez-Adan A, Jimenez A, Rojo C, et al. (2001). CMV-driven expression of green fluorescent protein (GFP) in male germ cells of transgenic mice and its effect on fertility. Int. J. Androl. 24: 300-305.

Whitelaw E, Sutherland H, Kearns M, Morgan H, et al. (2001). Epigenetic effects on transgene expression. Methods Mol. Biol. 158: 351-368.

Yang P, Wang J, Gong G, Sun X, et al. (2008). Cattle mammary bioreactor generated by a novel procedure of transgenic cloning for large-scale production of functional human lactoferrin. PLoS One 3: e3453. 